\title{
RÉSUMÉS - SOMMAIRE DES DONNÉES ACTUELLES SUR LE RISQUE DE TRANSMISSION DU VIH
}

\author{
PAQUETTE D, DEMERS A, GALE-ROWE M, WONG T
}

MOTS-CLÉS : VIH/SIDA, TRANSMISSION

\section{Résumé structuré}

\section{CONTEXTE :}

Les connaissances sur le risque de transmission du VIH ont évolué au cours de la dernière décennie à mesure que les données sur l'effet des cofacteurs biologiques et comportementaux, tels que la charge virale, ont été révélées. Un examen exhaustif des données sur le risque de la transmission du VIH a été mené.

\section{MÉTHODOLOGIE :}

Une recherche de la documentation publiée entre janvier 2001 et mai 2012 a été réalisée. Elle portait essentiellement sur les analyses systématiques, les méta-analyses et les revues narratives. Dans le cas des thèmes pour lesquels aucune analyse n'existait, des études de recherche primaire ont été incluses.

\section{RÉSULTATS :}

Les estimations du risque de transmission sexuelle du $\mathrm{VIH}$, par relation sexuelle, varient de $0,5 \%$ à $3,38 \%$ (estimations des valeurs moyennes : de 1,4\% à 1,69\%) pour les relations sexuelles anales réceptives; de $0,06 \%$ à $0,16 \%$ pour les relations sexuelles anales insertives; de $0,08 \%$ à $0,19 \%$ pour les relations sexuelles vaginales réceptives; et de $0,05 \%$ à $0,1 \%$ environ pour les relations sexuelles vaginales insertives. Chez les personnes qui s'injectent des drogues, on a estimé que le risque de transmission avec une aiguille contaminée, par injection, se situait entre $0,7 \%$ et $0,8 \%$. Un certain nombre de facteurs influent sur le risque, notamment la charge virale, la présence d'autres infections transmissibles sexuellement et la circoncision masculine.

\section{CONCLUSIONS :}

Pour chaque voie de transmission, les estimations du risque varient considérablement, probablement en raison du rôle de cofacteurs comportementaux et biologiques. La charge virale semble être un facteur prédictif de la transmission important, indépendamment de la voie de transmission. Toutefois, des données indiquent que la charge virale n'est pas le seul déterminant et que certains cofacteurs jouent un rôle dans l'augmentation ( $p$. ex. infections transmissibles sexuellement) ou la diminution (p. ex. circoncision masculine) du risque de transmission.

\section{Introduction}

Les connaissances sur le risque de transmission du VIH et les cofacteurs qui influencent le risque, particulièrement la charge virale et son rôle dans la transmission du VIH, évoluent. Cette information est importante pour les professionnels de la santé qui procèdent à des évaluations du risque et donnent du counseling. Elle sert aussi de base à une meilleure compréhension des nouvelles approches de prévention du VIH. En 2011, environ $84 \%$ des nouvelles infections au VIH étaient attribuables à la transmission sexuelle ( $47 \%$ chez les hommes ayant des relations sexuelles avec d'autres hommes; $17 \%$ chez les hétérosexuels de régions endémiques; $20 \%$ chez les hétérosexuels de régions non endémiques), et $14 \%$ des nouvelles infections concernaient des utilisateurs de drogues injectables (1). 
Il est essentiel de comprendre les déterminants biologiques de la transmission du VIH afin de pouvoir faire des prévisions sur la propagation potentielle de l'infection au VIH au sein d'une population, diriger des stratégies de prévention pertinentes et d'évaluer le risque d'infection d'une personne ayant été exposée au virus. Nos connaissances actuelles sur la transmission du VIH proviennent de divers types de données, y compris des études sur les animaux, des études d'observation, des essais contrôlés randomisés et des examens systématiques.

Le présent document est un sommaire des données actuelles sur la transmission sexuelle et la transmission associée à l'utilisation de drogues injectables et d'autres drogues, soit les deux voies de transmission les plus courantes au Canada. Un examen des données sur le risque de transmission verticale, en plus d'une description plus détaillée des risques associés à la transmission sexuelle et la transmission par l'usage de drogues, se trouvent dans le document intégral (2).

\section{Méthodologie}

Une recherche a été réalisée dans les bases de données Scopus, Embase et CINAHL; elle a été limitée aux articles publiés en anglais et en français de 2001 à 2012. Cette recherche était axée sur les analyses systématiques. Lorsqu'aucune analyse n'existait, des études de recherche primaire ont été incluses. Des études clés ou des publications souvent citées n'ayant pas été publiées au cours de la période visée ont également été incluses.

On a utilisé les termes de recherche suivants : (VIH ou « virus de l'immunodéficience humaine ») et (transmission ET [probabilité OU taux OU risque]) OU (par ET contact) OU (par ET relation) OU infectivité OU infectiosité OU transmissibilité, ainsi que des termes clés propres à chaque thème abordé dans le cadre de cette analyse.

\section{Résultats}

\section{TRANSMISSION PAR VOIE SEXUELLE}

Même si la quantification du risque par relation sexuelle est difficile, toutes les études signalent systématiquement que le risque associé aux relations sexuelles anales est plus élevé que celui des relations sexuelles vaginales, lesquelles sont associées à un risque plus élevé que celui des relations sexuelles orales. Il existe également un risque accru associé aux relations sexuelles réceptives (vaginales et anales) comparativement aux relations sexuelles insertives (Tableau 1).

Les estimations du risque de transmission du $\mathrm{VIH}$, par relation sexuelle, varient de $0,5 \%$ à 3,38 \% pour les relations sexuelles anales réceptives (3-6) et de $0,06 \%$ à $0,16 \%$ pour les relations sexuelles anales insertives (68). Bien que la plupart de ces estimations soient basées sur des études menées auprès d'hommes ayant des relations sexuelles avec des hommes, le risque associé aux relations sexuelles anales paraît semblable dans les populations hétérosexuelles (4).

Les estimations du risque de transmission du VIH pour les relations sexuelles vaginales réceptives (d'un homme à une femme) varient de $0,08 \%$ à $0,19 \%(5,6,9)$ et de $0.05 \%$ à $0,1 \%$ pour les relations sexuelles vaginales insertives (d'une femme à un homme) $(6,9)$.

Une méta-analyse laisse envisager une probabilité de transmission faible, mais non nulle associée aux relations sexuelles orales non protégées (pénis-bouche ou vagin-bouche) (10). L'éjaculation et la présence d'ulcérations buccales et d'infections transmissibles sexuellement dans l'oropharynx augmentent le risque de transmission au partenaire réceptif durant les relations sexuelles orales $(10,11)$. 


\section{CHARGE VIRALE}

Le plus puissant facteur prédictif de la transmission sexuelle du VIH est la charge virale plasmatique (12). Chaque augmentation de la charge virale plasmatique par un facteur de 10 est associée à un accroissement du risque relatif de transmission de 2,9 par contact sexuel (9). Le recours au traitement antirétroviral hautement actif, qui permet de réduire la charge virale, est associé à une baisse de $96 \%$ du nombre de transmissions connexes dans un essai contrôlé randomisé auprès de couples sérodiscordants (dont la plupart étaient hétérosexuels) (13). À l'heure actuelle, il n'est pas clair si un seuil de charge virale existe en dessous duquel il ne se produit pas de transmission. De plus, on sait peu de choses concernant l'impact de la charge virale sur le risque de transmission par relations sexuelles anales. Il est possible que le degré de réduction du risque associé au traitement antirétroviral hautement actif ne soit pas aussi efficace pour cette voie de transmission à risque plus élevé. Les résultats des études continues (PARTNER et Opposites Attract) seront utiles pour répondre à cette question.

II est probable que la charge virale plasmatique soit utilisée comme mesure indirecte de la charge virale du VIH dans les sécrétions génitales (14), ce qui joue un rôle majeur dans la transmission (15-17). Il a été conclu que les infections transmissibles sexuellement concomitantes augmentent la présence du VIH dans les voies génitales (1517). Bien qu'il ait été constaté que le traitement antirétroviral hautement actif inhibe la réplication virale dans les voies génitales, la non-observance a été associée à une présence persistante du virus dans les voies génitales (16). De plus, la présence du virus dans les voies génitales a même été constatée chez les personnes avec charge virale plasmatique non détectable $(18,19)$. Les conséquences de cette constatation sur le risque de transmission ne sont pas claires actuellement.

Les infections aux stades primaire (précoce) et tardif sont caractérisées par une charge virale élevée dans le plasma et dans les sécrétions génitales $(20,21)$. Dans le cas des primo-infections, cela s'explique par un degré de réplication virale élevé avant que le système immunitaire ait eu le temps de réagir (20). Les personnes qui sont au stade primaire de l'infection peuvent également présenter d'autres facteurs de risque ayant entraîné l'infection au $\mathrm{VIH}$ (22). Malgré la charge virale élevée au stade tardif de l'infection, on s'attend à ce que la contribution de l'infection à ce stade aux épidémies de VIH soit limitée, car les personnes atteintes de l'infection au stade tardif signalent des relations sexuelles moins fréquentes et moins de partenaires (21).

\section{INFECTIONS TRANSMISSIBLES SEXUELLEMENT (ITS)}

Des études d'observation ont montré que les infections transmissibles sexuellement étaient associées à une sensibilité accrue au VIH (23). Plusieurs examens systématiques d'études d'observation de qualité supérieure ont révélé que la présence d'ITS augmentait la sensibilité au VIH d'un facteur de 2 à 4 . Cet effet a été observé à la fois chez les hommes et chez les femmes, particulièrement dans les cas de virus de l'herpès simplex de type 2, de syphilis, de gonorrhée, de chlamydia, de trichomonas, et d'une exposition classée comme " toute ITS », " ulcérations génitales » et "ITS non ulcéreuses » (24-26). Des études d'observation plus récentes ont également montré que la présence du virus du papillome humain (VPH) était associée à l'acquisition du VIH chez les femmes, les hommes hétérosexuels et les hommes ayant des relations sexuelles avec des hommes $(27,28)$.

Des études révèlent que les infections transmissibles sexuellement sont aussi liées à un risque d'infectiosité accru. La majorité des données sur cette relation proviennent de méthodes indirectes, comme des études cliniques portant sur les mécanismes biologiques possibles qui sous-tendent le lien. Peu d'études d'observation ont examiné cette association (29). Cependant, un examen systématique de deux études a révélé que les ulcérations génitales et la syphilis augmentent de façon importante le risque de transmission du VIH (de 2 à 3 fois) (24).

Contrairement aux résultats des études d'observation, les résultats d'essais contrôlés randomisés portant sur l'effet du traitement des infections transmissibles sexuellement sur le risque de transmission du VIH étaient équivoques. Les résultats de neuf essais ont été publiés jusqu'à maintenant : six ont évalué les effets du traitement des infections transmissibles sexuellement guérissables et trois, les effets du traitement suppressif de l'herpès (30). L'essai Mwanza (Tanzanie) est le seul essai à avoir constaté un effet important, soit une réduction de $40 \%$ de l'incidence du $\mathrm{VIH}$ grâce à des services améliorés de traitement des infections transmissibles sexuellement (31). Les résultats équivoques peuvent s'expliquer par le type d'épidémie sévissant dans la collectivité (30). Dans le contexte d'une épidémie concentrée, comme c'est le cas dans l'essai Mwanza, les infections transmissibles sexuellement traitables 
peuvent constituer un cofacteur de transmission du VIH, ce qui n'est peut-être pas le cas dans le contexte d'épidémies généralisées des autres essais $(30,32)$. L'observance sous-optimale du traitement suppressif du virus de l'herpès simplex (VHS) et un manque de pouvoir statistique des études peuvent être à l'origine de l'incapacité des essais sur le traitement du VHS à montrer un effet important $(23,30)$.

\section{HOMMES NON CIRCONCIS}

Les trois essais contrôlés randomisés qui ont examiné l'effet de la circoncision chez l'homme ont tous indiqué une réduction du risque d'acquisition du VIH de l'ordre de $50 \%$ à $60 \%$ (33). Toutefois, il existe peu de données épidémiologiques montrant que la circoncision permet de réduire le risque de transmission aux partenaires de sexe féminin par les hommes circoncis (34) ou de prévenir le VIH chez les hommes ayant des relations sexuelles avec des hommes, à l'exception peut-être des hommes indiquant qu'ils jouent principalement le rôle du partenaire insertif (35).

\section{TRANSMISSION ASSOCIÉE À L'UTILISATION DE DROGUES}

\section{UTILISATION DE DROGUES INJECTABLES}

La probabilité de transmission du VIH par injection avec une aiguille ou une seringue contaminée a été estimée indirectement à l'aide de modèles mathématiques, en raison des difficultés de mesurer de façon précise le nombre d'expositions (c.-à-d. le nombre de fois qu'une aiguille ou seringue d'une personne séropositive a été partagée) et des autres facteurs de risque, tels que la charge virale. D'après ces modèles, la probabilité d'infection par injection avec une aiguille ou seringue contaminée serait de $0,67 \%$ à $0,84 \%(36,37)$. Tout comme dans le cas des estimations du risque de transmission sexuelle, ces mesures sommaires peuvent induire en erreur, car elles ne tiennent pas compte de l'hétérogénéité possible du risque de transmission par injection (37).

Un certain nombre d'études d'observation ont comparé le risque lié au partage d'aiguilles et de seringues par rapport au non-partage du matériel, et ce, dans les cas où l'état sérologique du partenaire d'injection n'était pas connu. Malgré le manque d'uniformité dans la mesure du partage des aiguilles et des seringues, les études épidémiologiques qui portaient sur le risque de transmission du $\mathrm{VIH}$ associé au partage des aiguilles et des seringues ont systématiquement conclu qu'il existait une relation positive. Dans les études de cohorte menées au Canada, les personnes qui partageaient des aiguilles et des seringues couraient un risque de 1,5 à 5,9 fois plus élevé de séroconversion $(38,39)$.

Des études laissent entendre que le partage du matériel d'injection auxiliaire (p. ex. partager l'eau, les contenants pour chauffer ou les filtres) augmente aussi le risque de transmission du VIH. Dans une étude menée en laboratoire, I'ADN du VIH a été détecté dans l'attirail d'injection, recueilli dans des piqueries à Miami (40). De plus, quelques études d'observation ont montré un lien entre le partage du matériel de préparation de la drogue et la transmission du VIH $(41,42)$.

\section{CHARGE VIRALE}

Il existe peu d'études d'observation de bonne qualité sur l'association entre la charge virale et le risque de transmission chez les utilisateurs de drogues injectables. Pour les utilisateurs de drogues injectables qui reçoivent le traitement antirétroviral hautement actif, le degré de réduction de l'infectiosité n'est pas connu. Des charges virales plasmatiques plus élevées ont été observées lors des éclosions d'infections à VIH chez ces personnes (43, 44). De plus, la charge virale dans la communauté des utilisateurs de drogues injectables était liée à l'incidence du VIH à Vancouver (45). La charge virale dans la communauté est la moyenne ou le total des mesures de la charge virale d'une population (46). La charge virale dans la communauté est une mesure globale, de sorte que tout lien établi à l'aide de cette mesure à l'échelle du groupe peut faire l'objet d'un sophisme écologique (c.-à-d. un lien entre des mesures globales ne constitue pas nécessairement une relation de cause à effet à l'échelle individuelle) (46). 


\section{TRANSMISSION SEXUELLE CHEZ LES UTILISATEURS DE DROGUES INJECTABLES}

Bien que le partage du matériel d'injection soit, principalement, à l'origine de l'épidémie de VIH chez les utilisateurs de drogues injectables, on a remarqué, au cours des dix dernières années, une diminution du partage des seringues. Des études ont montré que, après la prise en compte des comportements d'injection, la transmission sexuelle constituait une voie de transmission importante au sein de ce groupe (47). La séroconversion VIH chez les utilisateurs de drogues injectables est associée de façon indépendante au fait d'avoir un partenaire sexuel séropositif pour le $\mathrm{VIH}$ et d'adopter des comportements sexuels à risque (p. ex. partenaires sexuels multiples, travail dans l'industrie du sexe et utilisation irrégulière du condom) $(48,49)$.

\section{UTILISATION DE DROGUES NON INJECTABLES}

L'utilisation de certaines drogues non injectables a été signalée comme un facteur de risque indépendant de transmission du VIH. Le fait de fumer du crack (isolément) ainsi que la prise d'amphétamines ont été décrits comme des facteurs de risque indépendants de séropositivité qui entraînent un risque de 2 à 3 fois plus élevé $(50,51)$. Parmi les limites importantes de ces études, notons les résultats fondés sur des données autodéclarées et la difficulté de tenir compte des facteurs de confusion.

II existe peu de données sur les mécanismes de transmission du VIH par la consommation de drogues par reniflement ou inhalation seulement. On a proposé que le partage de l'attirail, comme des pailles, des billets de banque et des pipes à crack ou des tubes, pouvait constituer une voie de transmission. Les coupures, les éruptions vésiculaires et les plaies sur les lèvres des fumeurs de crack peuvent faciliter la transmission orale du VIH (52-54); les données sur cette relation de cause à effet s'accumulent, mais sont encore peu nombreuses (11).

\section{Conclusion}

Le risque de transmission du VIH est complexe et dépend de différents cofacteurs comportementaux et biologiques. Il est toujours difficile de quantifier précisément le risque de transmission associé à un facteur en particulier. Cependant, en ce qui concerne la transmission sexuelle, les relations sexuelles anales réceptives non protégées comportent le plus grand risque. La charge virale plasmatique semble être un facteur prédictif de la transmission important pour toutes les voies de transmission. Toutefois, bien que la charge virale soit un facteur clé de la transmission ou de l'absence de transmission du $\mathrm{VIH}$, les données indiquent qu'il ne s'agit pas du seul déterminant et que d'autres cofacteurs jouent un rôle dans l'augmentation ou la diminution du risque de transmission. Cette analyse des données montre que nous enrichissons et perfectionnons nos connaissances sur le risque de transmission du VIH, de même que sur les cofacteurs comportementaux et biologiques qui influent sur ce risque.

\section{Remerciements}

Mille mercis à Chris Archibald, à Christopher Boodram, à Katherine Dinner, à Katie Freer, à Brian Gottheil, à Ping Yan et à Ameeta Singh pour avoir examiné le document intégral et présenté leurs commentaires.

\section{Déclaration de conflit d'intérêts}

II n'y a aucun conflit d'intérêts à déclarer.

\section{Financement}

Ce travail a été appuyé par l'Agence de la santé publique du Canada. 
TABLEAU 1. ESTIMATIONS DU RISQUE DE TRANSMISSION PAR RAPPORT SEXUEL ET PAR INJECTION

Voie de transmission

Relations sexuelles anales réceptives

Relations sexuelles anales insertives

Relations sexuelles anales insertives

Relations sexuelles vaginales réceptives

Relations sexuelles vaginales insertives

Relations sexuelles orales

Partage d'aiguilles et de seringues contaminées
Probabilité de transmission

Références

\section{Références}

1. Agence de la santé publique du Canada. Résumé : Estimations de la prévalence et de l'incidence du VIH au Canada, 2011. Ottawa : Gouvernement du Canada; 2012.

2. Agence de la santé publique du Canada. Risque de transmission du VIH : Sommaire des données scientifiques. Ottawa : Gouvernement du Canada; 2012. http://publications.gc.ca/site/fra/434867/publication.html

3. Powers KA, Poole C, Pettifor AE, Cohen MS. Rethinking the heterosexual infectivity of HIV-1: A systematic review and meta-analysis. Lancet Infect Dis. 2008;8(9):553-63.

4. Baggaley RF, White RG, Boily MC. HIV transmission risk through anal intercourse: Systematic review, meta-analysis and implications for HIV prevention. Int J Epidemiol. 2010;39(4):1048-63.

5. Boily MC, Baggaley RF, Wang L, Masse B, White RG, Hayes RJ, et al. Heterosexual risk of HIV-1 infection per sexual act: Systematic review and meta-analysis of observational studies. Lancet Infect Dis. 2009;9(2):118-29.

6. Fox J, White PJ, Weber J, Garnett GP, Ward H, Fidler S. Quantifying sexual exposure to HIV within an HIVserodiscordant relationship: Development of an algorithm. AIDS. 2011;25(8):1065-82.

7. Vittinghoff E, Douglas J, Judson F, McKirnan D, Macqueen K, Buchbinder SR. Per-contact risk of human immunodeficiency virus transmission between male sexual partners. Am J Epidemiol. 1999;150(3):306-11.

8. Jin F, Jansson J, Law M, Prestage GP, Zablotska I, Imrie JCG, et al. Per-contact probability of HIV transmission in homosexual men in Sydney in the era of HAART. AIDS. 2010;24(6):907-13.

9. Hughes JP, Baeten JM, Lingappa JR, Magaret AS, Wald A, de Bruyn G, et al. Determinants of per-coital-act HIV-1 infectivity among african HIV-1-serodiscordant couples. J Infect Dis. 2012 February 01;205(3):358-65.

10. Baggaley RF, White RG, Boily MC. Systematic review of orogenital HIV-1 transmission probabilities. Int J Epidemiol. 2008;37(6):1255-65.

11. Campo J, Perea MA, Del Romero J, Cano J, Hernando V, Bascones A. Oral transmission of HIV, reality or fiction? an update. Oral Dis. 2006;12(3):219-28.

12. Fox J, Fidler S. Risk of HIV transmission in discordant partners. J HIV Ther. 2007;12(2):48-53.

13. Cohen MS, Chen YQ, McCauley M, Gamble T, Hosseinipour MC, Kumarasamy N, et al. Prevention of HIV-1 infection with early antiretroviral therapy. N Engl J Med. 2011;365(6):493-505.

14. Cohen MS, Gay C, Kashuba AD, Blower S, Paxton L. Narrative review: Antiretroviral therapy to prevent the sexual transmission of HIV-1. Ann Intern Med. 2007;146(8):591-601. 
15. Anderson BL, Cu-Uvin S. Determinants of HIV shedding in the lower genital tract of women. Curr Infect Dis Rep. 2008;10(6):505-11.

16. Kalichman SC, Di Berto G, Eaton L. Human immunodeficiency virus viral load in blood plasma and semen: Review and implications of empirical findings. Sex Transm Dis. 2008;35(1):55-60.

17. Spinillo A, Gardella B, Zanchi S, Roccio M, Preti E. Determinants of genital shedding of human immunodeficiency virus: A review. Curr Women's Health Rev. 2008;4(2):118-23.

18. Politch JA, Mayer KH, Welles SL, O'brien WX, Xu C, Bowman FP, et al. Highly active antiretroviral therapy does not completely suppress HIV in semen of sexually active HIV-infected men who have sex with men. AIDS. 2012 Mar 23.

19. Cu-Uvin S, DeLong AK, Venkatesh KK, Hogan JW, Ingersoll J, Kurpewski J, et al. Genital tract HIV-1 RNA shedding among women with below detectable plasma viral load. AIDS. 2010;24(16):2489-97.

20. Miller WC, Rosenberg NE, Rutstein SE, Powers KA. Role of acute and early HIV infection in the sexual transmission of HIV. Curr Opin HIV AIDS. 2010;5(4):277-82.

21. Wawer MJ, Gray RH, Sewankambo NK, Serwadda D, Li X, Laeyendecker O, et al. Rates of HIV-1 transmission per coital act, by stage of HIV-1 infection, in Rakai, Uganda. J Infect Dis. 2005;191(9):1403-9.

22. Dosekun O, Fox J. An overview of the relative risks of different sexual behaviours on HIV transmission. Current Opinion in HIV and AIDS. 2010;5(4):291-7.

23. Ward H, Rönn M. Contribution of sexually transmitted infections to the sexual transmission of HIV. Current Opinion in HIV and AIDS. 2010;5(4):305-10.

24. Røttingen JA, Cameron WD, Garnett GP. A systematic review of the epidemiologic interactions between classic sexually transmitted diseases and HIV: How much really is known? Sex Transm Dis. 2001;28(10):579-97.

25. Freeman EE, Weiss HA, Glynn JR, Cross PL, Whitworth JA, Hayes RJ. Herpes simplex virus 2 infection increases HIV acquisition in men and women: Systematic review and meta-analysis of longitudinal studies. AIDS. 2006;20(1):73-83.

26. Sexton J, Garnett G, Rottingen JA. Metaanalysis and metaregression in interpreting study variability in the impact of sexually transmitted diseases on susceptibility to HIV infection. Sex Transm Dis. 2005;32(6):351-7.

27. Auvert B, Marais D, Lissouba P, Zarca K, Ramjee G, Williamson AL. High-risk human papillomavirus is associated with HIV acquisition among south african female sex workers. Infect Dis Obstet Gynecol. 2011;2011:692012.

28. Smith JS, Moses S, Hudgens MG, Parker CB, Agot K, Maclean I, et al. Increased risk of HIV acquisition among kenyan men with human papillomavirus infection. J Infect Dis. 2010;201(11):1677-85.

29. Galvin SR, Cohen MS. The role of sexually transmitted diseases in HIV transmission. Nat Rev Microbiol. 2004;2(1):33-42.

30. Hayes R, Watson-Jones D, Celum C, van de Wijgert J, Wasserheit J. Treatment of sexually transmitted infections for HIV prevention: End of the road or new beginning? AIDS. 2010;24(SUPPL. 4):S15-26.

31. Grosskurth H, Mosha F, Todd J, Mwijarubi E, Klokke A, Senkoro K, et al. Impact of improved treatment of sexually transmitted diseases on HIV infection in rural tanzania: Randomised controlled trial. Lancet. 1995;346(8974):530-6.

32. Barnabas RV, Wasserheit JN. Riddle of the sphinx revisited: The role of STDs in HIV prevention. Sex Transm Dis. 2009;36(6):365-7.

33. Siegfried N, Muller M, Deeks JJ, Volmink J. Male circumcision for prevention of heterosexual acquisition of HIV in men. Cochrane Database Syst Rev. 2009(2).

34. Weiss HA, Hankins CA, Dickson K. Male circumcision and risk of HIV infection in women: A systematic review and metaanalysis. Lancet Infect Dis. 2009;9(11):669-77.

35. Millett GA, Flores SA, Marks G, Reed JB, Herbst JH. Circumcision status and risk of HIV and sexually transmitted infections among men who have sex with men: A meta-analysis. JAMA. 2008;300(14):1674-84.

36. Kaplan EH, Heimer R. A model-based estimate of HIV infectivity via needle sharing. J Acquir Immune Defic Syndr. 1992;5(11):1116-8. 
37. Hudgens MG, Longini Jr. IM, Halloran ME, Choopanya K, Vanichseni S, Kitayaporn D, et al. Estimating the transmission probability of human immunodeficiency virus in injecting drug users in thailand. Appl Statist. 2001;50(1):1-14.

38. Miller CL, Kerr T, Frankish JC, Spittal PM, Li K, Schechter MT, et al. Binge drug use independently predicts HIV seroconversion among injection drug users: Implications for public health strategies. Subst Use Misuse. 2006;41(2):199210.

39. Bruneau, J., Daniel, M., Abrahamowicz, M., Zang, G., Lamothe, F., Vincelette, J. Trends in human immunodeficiency virus incidence and risk behavior among injection drug users in Montreal, Canada: A 16-year longitudinal study. Am J Epidemiol. 2011;173(9):1049-58.

40. Shah SM, Shapshak P, Rivers JE, Stewart RV, Weatherby NL, Xin KQ, et al. Detection of HIV-1 DNA in needle/syringes, paraphernalia, and washes from shooting galleries in Miami: A preliminary laboratory report. J Acquir Immune Defic Syndr Hum Retrovirol. 1996 Mar 1;11(3):301-6.

41. Brogly SB, Bruneau J, Vincelette J, Lamothe F, Franco EL. Risk behaviour change and HIV infection among injection drug users in montreal. AIDS. 2000 Nov 10;14(16):2575-82.

42. Zhang Y, Shan H, Trizzino J, Ruan Y, Beauchamp G, Masse B, et al. Demographic characteristics and risk behaviors associated with HIV positive injecting drug users in Xinjiang, China. J Infect. 2007;54(3):285-90.

43. Hu DJ, Subbarao S, Vanichseni S, Mock PA, van Griensven F, Nelson R, et al. Higher viral loads and other risk factors associated with HIV-1 seroconversion during a period of high incidence among injection drug users in bangkok. $J$ Acquir Immune Defic Syndr. 2002 Jun 1;30(2):240-7.

44. Kivelä PS, Krol A, Salminen MO, Geskus RB, Suni JI, Anttila VJ, et al. High plasma HIV load in the CRF01-AE outbreak among injecting drug users in Finland. Scand J Infect Dis. 2005;37(4):276-83.

45. Wood E, Kerr T, Marshall BD, Li K, Zhang R, Hogg RS, et al. Longitudinal community plasma HIV-1 RNA concentrations and incidence of HIV-1 among injecting drug users: Prospective cohort study. BMJ. 2009;338.

46. Castel AD, Befus M, Willis S, Griffin A, West T, Hader S, et al. Use of the community viral load as a population-based biomarker of HIV burden. AIDS. 2012 Jan 28;26(3):345-53.

47. Des Jarlais DC, Arasteh K, McKnight C, Hagan H, Perlman DC, Semaan S. Associations between herpes simplex virus type 2 and HCV with HIV among injecting drug users in new york city: The current importance of sexual transmission of HIV. Am J Public Health. 2011;101(7):1277-83.

48. Strathdee SA, Galai N, Safaiean M, Celentano DD, Vlahov D, Johnson L, et al. Sex differences in risk factors for hiv seroconversion among injection drug users: A 10-year perspective. Arch Intern Med. 2001 May 28;161(10):1281-8.

49. Bacon O, Lum P, Hahn J, Evans J, Davidson P, Moss A, et al. Commercial sex work and risk of HIV infection among young drug-injecting men who have sex with men in San Francisco. Sex Transm Dis. 2006;33(4):228-34.

50. McCoy CB, Lai S, Metsch LR, Messiah SE, Zhao W. Injection drug use and crack cocaine smoking: Independent and dual risk behaviors for HIV infection. Ann Epidemiol. 2004 Sep;14(8):535-42.

51. DeBeck K, Kerr T, Li K, Fischer B, Buxton J, Montaner J, et al. Smoking of crack cocaine as a risk factor for HIV infection among people who use injection drugs. CMAJ. 2009;181(9):585-9.

52. Faruque S, Edlin BR, McCoy CB, Word CO, Larsen SA, Schmid DS, et al. Crack cocaine smoking and oral sores in three inner-city neighborhoods. J Acquir Immune Defic Syndr Hum Retrovirol. 1996 Sep;13(1):87-92.

53. Porter J, Bonilla L, Drucker E. Methods of smoking crack as a potential risk factor for HIV infection: Crack smokers' perception and behavior. Contemp Drug Probl. 1997;24:319-48.

54. Porter J, Bonilla L. Crack users' cracked lips: An additional HIV risk factor. Am J Public Health. 1993 Oct;83(10):1490-1. 